\title{
Videoactivismo y trabajo por proyectos: una experiencia de Educación para la Ciudadanía Mundial en Colombia
}

\section{Resumen}

Nicolás J. C. Aguilar Forero ${ }^{1}$

ORCID: https://orcid.org/0000-0002-9181-0281

La Educación para la Ciudadanía Mundial es un concepto y estrategia educativa emergente que distintos gobiernos y organizaciones internacionales vienen promoviendo como respuesta a los grandes problemas que afectan a la humanidad: pobreza, desigualdad, violencias, devastación ambiental, entre otros. Sin embargo, en la actualidad no hay ningún acuerdo acerca de qué significa ser un ciudadano del mundo y qué características deberían tener las estrategias de Educación para la Ciudadanía Mundial en contextos específicos. En este marco, el presente artículo expone los resultados de una investigación que buscó comprender cómo el activismo mediado por tecnologías digitales puede contribuir a la Educación para la Ciudadanía Mundial. El estudio se desarrolló durante al año 2017 en dos instituciones educativas de la ciudad de Bogotá, Colombia, que promueven la apropiación crítica de nuevos medios y tecnologías: la Escuela Mediática y el Colegio Venecia. A partir de una Investigación Acción Participativa y de la combinación de técnicas como la observación participante, las entrevistas semiestructuradas y el análisis de contenido, se identificó que los estudiantes de estas instituciones educativas, a través del videoactivismo y del trabajo por proyectos, promueven la reflexión y generación de conciencia en torno a temas que, como el cuidado del medio ambiente, conciernen a la humanidad y son muy cercanos a sus sensibilidades juveniles.

\section{Palabras clave}

Educación para la ciudadanía mundial - Videoactivismo - Trabajo por proyectos.

1- Universidad de los Andes, Bogotá, Colombia. Contacto: nj.aguilar1902@uniandes.edu.co

(cc) (P) DOl: http://dx.doi.org/10.1590/\$1678-4634201945191009

This content is licensed under a Creative Commons attribution-type BY-NC. 


\title{
Video-activism and project-based assignments: an experience of Global Citizenship Education in Colombia
}

\begin{abstract}
Global Citizenship Education is an emerging educational concept and strategy that different governments and international organizations have been promoting in response to the great problems that affect humanity including poverty, inequality, violence, and environmental devastation. However, at present there is no agreement about what it means to be a citizen of the world and what features Global Citizenship Education strategies should take in specific contexts. This article presents the results of a study that sought to understand how activism mediated by digital technologies can contribute to Global Citizenship Education. The study was developed in 2017 in two educational institutions of Bogotá, Colombia, which promote critical appropriation of new media and technologies: Escuela Mediática and Colegio Venecia. Based on a Participatory Action Research and the combination of techniques such as participant observation, semi-structured interviews and content analysis, it was identified that students from these educational institutions, by using video-activism and project work, promote reflection and raise awareness about issues that, such as caring for the environment, concern humanity and are very close to their youthful sensitivities.
\end{abstract}

\section{Keywords}

Global Citizenship Education - Video-activism - project-based assignments.

\section{Introducción}

La Educación para la Ciudadanía Mundial (en adelante ECM) es un movimiento de educación global que busca promover la solidaridad y la construcción de una ciudadanía comprometida con la lucha en contra de la pobreza, la injusticia, la exclusión, las violencias y la devastación ambiental. Se trata de una estrategia educativa emergente con la que se espera responder a las necesidades de las sociedades actuales, bajo la premisa de que la educación no puede quedarse en una mirada estrecha y localista, sino que debe asumir una dimensión global con el propósito de formar a las personas y promover su desenvolvimiento en un mundo atravesado por problemáticas con orígenes diversos (locales, nacionales, globales) y explicaciones complejas (BONI; HOFMAN-PINILLA; SOW, 2012).

Si bien la ECM ha sido impulsada por distintos gobiernos y organizaciones con incidencia internacional como la Unesco (2016), en la actualidad no hay ningún acuerdo acerca de qué significa ser un ciudadano del mundo y qué características deberían tener 
las estrategias de ECM en contextos específicos. Sin embargo, entre los numerosos debates y agendas, sobresalen tres enfoques ampliamente discutidos en la literatura académica: 1) Neoliberal. La ECM desde este enfoque opera como un medio para proveer a los estudiantes de las habilidades necesarias para adaptarse a un mundo globalizado, hiperconectado y basado en una economía de mercado/consumo. 2) Humanista. Se centra en la promoción de los derechos humanos como valores universales y en una ECM que propicie la responsabilidad de los sujetos de cara a la construcción de un mundo más equitativo y pacífico. 3) Crítico. Desde este enfoque el objetivo fundamental de la ECM consiste en confrontar la injusticia social y las inequidades globales/locales que las anteriores aproximaciones, aunque intenten evitarlo, terminan por reproducir (SHULTZ, 2007; ANDREOTTI, 2006; CHO; MOSSELSON, 2017; PAIS; COSTA, 2017).

La relación entre estos enfoques, desde luego, ha sido mucho más compleja de lo que se alcanza a expresar con la enumeración anterior. Algunos autores han mostrado que tales aproximaciones en la práctica se sobreponen y han identificado, incluso, que muchas de las agendas de ECM que se promueven desde un enfoque crítico en realidad no se oponen sino que se complementan con un enfoque neoliberal (CAMICIA; FRANKLIN, 2011; PAIS; COSTA, 2017). De igual forma, esta categorización en tres grandes perspectivas de ECM corre el riesgo de homogenizar la diversidad al interior de cada uno de estos discursos o perspectivas. Por ejemplo, dentro del enfoque crítico de ECM se identifican al menos tres aproximaciones distintas: una basada en la pedagogía crítica y la educación popular (VEUGELERS, 2007; CATALANO, 2013; REILLY; NIENS, 2014; TRUONG-WHITE; MCLEAN, 2015); un segundo enfoque que cuestiona la ECM como concepto y estrategia educativa emergente, de manera que su crítica no se despliega al interior de la ECM sino en contra de esta (WATSON, 2013; PAIS; COSTA, 2017; JOOSTE; HELETA, 2017); y un tercer enfoque decolonial que, si bien también bebe de la pedagogía crítica (por eso algunos autores lo asocian al enfoque crítico y no lo conciben como un enfoque independiente), se soporta fundamentalmente en las propuestas del grupo modernidad/colonialidad y en especial de autores como Walter Mignolo, Arturo Escobar, Aníbal Quijano, Ramón Grosfoguel, Enrique Dussel, Catherine Walsh y Nelson Maldonado-Torres (ANDREOTTI, 2011; BALARIN, 2011; NIETO, 2017).

Lo cierto es que, desde esta última aproximación, a lo largo del año 2017 se puso en marcha una investigación con el propósito de comprender cómo el activismo mediado por tecnologías digitales podría contribuir a la ECM. Este estudio se basó en una experiencia de intercambio y colaboración entre dos instituciones educativas que, en la ciudad de Bogotá, Colombia, promueven el uso y apropiación de tecnologías digitales como base para la comunicación, expresión y aprendizaje de los jóvenes. El trabajo se desarrolló en la Escuela Mediática, entidad de carácter privado que tiene como misión el desarrollo de experiencias alternativas de educación orientadas a la formación de ciudadanos críticos y a la superación de las pedagogías tradicionales (ESCUELA MEDIÁTICA, 2000). También tuvo lugar en el Colegio Venecia, institución educativa de carácter oficial que ofrece a sus estudiantes una educación media en la que la comunicación, la programación y el diseño web, ocupan un lugar central (LEÓN, 2017). Pese a que en ambos colegios la ECM era un tema que no se había trabajado previamente, fue bien recibido, entre otras cosas porque se identificó que podría articularse con facilidad a lo que ya se venía haciendo en los colegios con respecto 
a la educación para la ciudadanía y, particularmente, a sus propuestas pedagógicas basadas en la comunicación y la apropiación crítica de tecnologías digitales.

Aunque diversas investigaciones han analizado la interacción entre tecnologías digitales y diferentes estrategias educativas encaminadas a la generación de ciertos aprendizajes (ROMAN; MURILLO, 2014) o a la promoción del cambio social (LINDER et al., 2016; FULLAM, 2017; CHUNG; KIRBY, 2009; GLENN, 2015), cabe destacar que aún son escasos los estudios relacionados con los vínculos entre el activismo mediado por tecnologías digitales y la ECM. La presente investigación contribuye a llenar este vacío y evidencia que los estudiantes de las dos instituciones educativas mencionadas aportan a la ECM, a través del videoactivismo y el trabajo por proyectos. Asimismo, propone claves interesantes acerca de cómo desarrollar iniciativas de ECM desde un enfoque decolonial, sacando provecho de las herramientas tecnológicas y en diálogo con las sensibilidades juveniles.

\section{La ECM desde un enfoque decolonial}

Como ya se anticipó, la ECM no puede pensarse como un movimiento educativo homogéneo, pues se ha desplegado a través de diferentes agendas, discursos y apuestas. El discurso neoliberal, por ejemplo, se ha enfocado en el emprendimiento y el desarrollo individual, con el fin de preparar a las personas para que sean competitivas en un mundo ya existente, globalizado e interconectado. Como señalan Pais y Costa (2017) el propósito de este discurso no ha sido el de formar para el criticismo o la imaginación de alternativas al mundo actual, sino optimizar el sistema existente y desarrollar capacidades para que las personas puedan adaptarse mejor al mismo. En este marco los problemas, aunque son reconocidos, son percibidos como disfuncionalidades de un sistema que puede ser mejorado por sujetos cada vez más competitivos y emprendedores. La injusticia social, por ejemplo, es concebida como un problema que debe ser corregido para que el sistema funcione mejor y no como una condición inherente al mismo y que garantiza su reproducción.

Por su parte, el discurso humanista basado en los derechos humanos y en el intento de democratizar la sociedad, enfatiza en el rol de una ciudadanía activa y responsable capaz de reconocer los problemas mundiales y de trabajar conjuntamente para superarlos. No obstante, al no cuestionar de fondo las causas estructurales de los problemas sociales ligadas, entre otras, al capitalismo, el colonialismo y el heteropatriarcado (SANTOS, 2010), las estrategias de ECM desde este enfoque terminan alineadas con las del discurso neoliberal y en la práctica refuerzan el sistema que en apariencia esperan transformar (CHO; MOSSELSON, 2017). Como agregan Pais y Costa (2017), pese a la inclusión y democratización que promueve el discurso humanista y democrático de ECM, en última instancia la ECM sigue siendo un concepto muy local, restringido e incluso eurocéntrico, pues la mayor producción sobre el tema se encuentra en Estados Unidos, Reino Unido, Australia y Canadá, y ha sido generada por una élite blanca y liberal que pertenece a los países dominantes. De manera que, mientras se promueve una comunidad global, se beneficia en realidad a un grupo muy reducido.

Además de los cuestionamientos anteriores, autores como Jooste y Heleta (2017) han señalado que la paz, la sostenibilidad ambiental, la satisfacción de necesidades básicas y 
en general la supervivencia de la humanidad y de la vida en la tierra, no dependen de si somos o no ciudadanos del mundo. Dependen, por el contrario, de nuestra habilidad para persuadir a los países poderosos de cambiar una economía desigual, injusta y opresiva, junto con los sistemas políticos que la soportan, para que los países subalternizados puedan ser prósperos y tener condiciones de vida dignas. Por lo tanto, en lugar de títulos o conceptos de moda como el de ECM, promovidos por los países del norte y por los académicos de las universidades de orientación liberal (también del norte) que a última hora incluyen ideas del sur para darle legitimidad a sus discursos y parecer inclusivos, lo que necesitamos son sujetos comprometidos y activos en términos ciudadanos, que se preocupen por sus países, regiones y el mundo entero, y que piensen tanto local como globalmente (JOOSTE; HELETA, 2017).

Ahora bien, frente al enfoque neoliberal y humanista se han venido fortaleciendo otras agendas de ECM que si bien abandonan las posturas celebratorias y acríticas de la misma, al tiempo procuran no caer en posicionamientos que rechazan de tajo sus posibles contribuciones. Tal es el caso del trabajo de Vanessa de Oliveira Andreotti (2006; 2011), quien promueve una lecutra decolonial de la ECM, con el fin de reconocer las particularidades de los contextos concretos y evitar interpretaciones universalistas o asistencialistas que reproducen el triunfalismo de los países dominantes, convencidos de que son el centro del mundo y de que tienen la responsabilidad de ayudar al resto para que puedan llegar a ser desarrollados y para que sus ciudadanos devengan en ciudadanos del mundo (ANDREOTTI; PASHBY, 2013).

Teniendo en cuenta que el enfoque decolonial no es tampoco un enfoque homogéneo, en esta investigación se acoge específicamente la perspectiva de Andreotti (2011), en la que se identifican por lo menos cuatro características centrales de la ECM desde un enfoque decolonial: 1) procura no partir de un ideal normativo de ciudadano del mundo asociado a un conjunto de valores predeterminados y a las formas de vida dominantes en las sociedades liberales-occidentales. Lo anterior implica no asumir la universalidad de un sujeto considerado como ciudadano del mundo o potencial ciudadano del mundo, sino analizar en qué lugar o contexto está situado ese sujeto y cómo ha sido social e históricamente constituido en esa posición (ANDREOTTI, 2011); 2) reconoce y problematiza las geopolíticas del conocimiento, es decir, el lugar concreto desde donde se piensa, se produce conocimiento y se interviene en el mundo; 3) evita tomar por sentada la neutralidad y universalidad de otros discursos como el de los derechos humanos, y por el contrario reivindica su apertura y su posible enriquecimiento desde epistemologías alternativas a las de las sociedades liberales y occidentales dominantes (ANDREOTTI, 2006; SANTOS, 2010; NIETO, 2017).

Desde esta perspectiva, en lugar de imponer el discurso de ECM como el camino necesario y esencialmente positivo de la educación contemporánea para construir un mundo mejor (PAIS; COSTA, 2017), se propone conocer a profundidad los contextos en los que se trabaja y evitar caer en la implementación de acciones descontextualizadas. Siguiendo esta consideración, en el presente estudio se conocieron a profundidad las propuestas educativas de las dos instituciones educativas con las que se trabajó, y se identificó la importancia de la producción audiovisual en una de estas. Por tal motivo fue necesario 
apelar a conceptos emergentes y profundizar en la noción de videoactivismo, entendido como una de las modalidades del activismo mediado por herramientas tecnológicas. Dicha exploración no fue simple debido a que, como bien señalan Peña, Rodríguez y Sáez (2016), aunque hay una abundante literatura sobre activismo mediado por tecnologías, también llamado ciberactivismo (ENGUIX, 2016; GONZÁLEZ-LIZARRAGA; BECERRA-TRAVER; YANEZ-DÍAZ, 2016; VALENZUELA, 2013), esta contrasta con la poca sistematicidad de la reflexión académica sobre el videoactivismo digital.

Pese a lo anterior, para efectos de este trabajo se construye y comprende el videoactivismo como un conjunto de prácticas político-comunicativas desarrolladas por actores ajenos a las estructuras de poder dominantes, y cuya intencionalidad transformadora puede tener diferentes fines tácticos: contrainformar, generar conciencia y reflexión, formar, convocar para futuras acciones online u offline, articular la participación, documentar injusticias o construir sentido de pertenencia, compromiso e identidad colectiva (MATEOS; RAJAS, 2014; ASKANIUS, 2013; GARCÍA, 2014). Asimismo, el videoactivismo puede complementarse con el uso de redes sociales $u$ otras plataformas digitales y puede promoverse a través de diversos procesos educativos (PEÑA, RODRÍGUEZ; SÁEZ, 2016). Esto fue precisamente lo que se logró en el presente estudio: adelantar un proceso educativo en el que, por medio del videoactivismo y el trabajo por proyectos, se aportó a la ECM.

Para terminar esta sección, cabe destacar que al profundizar en las estrategias pedagógicas de ambos colegios se identificó también la importancia del trabajo por proyectos. Dicho trabajo se configura como una oportunidad e incluso como una alternativa frente a la tendencia identificada por algunos autores (MARSHALL, 2011; STANDISH, 2014; ENGEL; FUNDALINSKI; CANNON, 2016) a reducir la ECM a un conjunto normativo de valores o lineamientos que, inspirados en el pensamiento liberal-occidental, imponen lo que debe saber y saber hacer un ciudadano del mundo como resultado de cierta trayectoria educativa. Por el contrario, el trabajo por proyectos permite que emerjan comprensiones situadas acerca de lo que para los jóvenes significa ser un ciudadano del mundo, así como iniciativas y acciones colectivas para generar transformaciones globales vinculadas con preocupaciones locales. Asimismo, el trabajo por proyectos permite superar las fronteras disciplinarias y desplegar la creatividad de los estudiantes con base en orientaciones, pero no, en protocolos teórico-metodológicos cerrados y trazados de antemano. Esta es una de las características inherentes al trabajo por proyectos:

Los proyectos son expresión de creatividad y su sentido radica precisamente en la diversidad y creatividad ilimitada que los nutre. Esta diversidad de caminos puede apoyarse no solo en un pluralismo metodológico sino también en una apertura disciplinaria. Dicho de otro modo: la pedagogía por proyectos se mueve en la transdisciplinariedad. (IEIE, 2017, p. 409).

\section{Propuesta metodológica}

A lo largo del año 2017 se puso en marcha una Investigación-Acción Participativa (IAP) con el fin de generar conocimientos y prácticas pedagógicas desde la experiencia 
directa de los actores involucrados en el estudio. La IAP es una modalidad de investigación inscrita en un enfoque sociocrítico, motivo por el cual no solo apunta a generar comprensiones relevantes para los investigadores sino también transformaciones concretas de las prácticas sociales y de los contextos en los que se investiga. En este tipo de investigación, a través de la producción de conocimiento y del desarrollo de actividades planeadas, implementadas y evaluadas colectivamente, los actores involucrados participan como coinvestigadores y, al mismo tiempo, como agentes comprometidos con el cambio social (FALS BORDA, 2009) ${ }^{2}$. Aunque el grado de implicación entre los actores que participan del estudio puede variar de acuerdo con la etapa, los conocimientos previos o la disponibilidad de las partes, en este tipo de investigación cooperativa se busca equilibrar las relaciones de poder (BONI; HOFMAN-PINILLA; SOW, 2012) y confrontar la tradicional distinción entre sujetos y objetos de conocimiento. Desde esta perspectiva se adelantaron las siguientes tres fases que integraron la propuesta metodológica:

Fase 1: recuperación histórica y diseño metodológico. Luego de conformar un grupo de trabajo integrado por dos docentes de la Escuela Mediática, tres docentes del Colegio Venecia y tres investigadores de la Facultad de Educación de la Universidad de los Andes de Bogotá, se diseñaron e implementaron once entrevistas semiestructuradas con docentes y exalumnos de ambos colegios, con el propósito de comprender a profundidad el trabajo que en estos se venía desarrollando en la educación media (con jóvenes entre los 15 y 18 años de edad aproximadamente). A partir de estas entrevistas se reconstruyó la historia (trayectoria, aciertos y dificultades) del área de comunicación en la Escuela Mediática y del área denominada Educación Media Integral, del Colegio Venecia. También se hizo observación participante en cuatro clases de cada área con el fin de comprender las dinámicas y prácticas pedagógicas implementadas. Con esta base, se diseñaron conjuntamente una serie de acciones que permitieran responder la pregunta central del estudio, previamente acordada: ¿cómo a través del activismo mediado por tecnologías digitales se puede contribuir a la ECM?

Fase 2: implementación de acciones. Las acciones diseñadas e implementadas fueron las siguientes: 1) análisis de contenidos gráficos y audiovisuales producidos por los estudiantes de ambos colegios entre el año 2016 y el año 2017; 2) ocho talleres con 48 estudiantes de grado décimo y once (últimos grados de la educación media en Colombia) de la Escuela Mediática y ocho talleres con 40 estudiantes de grado once del Colegio Venecia; 3) un taller conjunto de intercambio de experiencias con participación de tres docentes y 50 estudiantes de ambos colegios; 4) un evento de socialización de resultados del trabajo desarrollado con participación de cuatro docentes, 50 estudiantes y cinco invitados externos. Cabe destacar que los 16 talleres, ocho en cada colegio, permitieron reflexionar acerca de lo que significa para los estudiantes ser un ciudadano del mundo, y analizar las posibilidades del activismo mediado por tecnologías de cara a la superación de una serie de problemáticas mundiales identificadas por ellos. Estos talleres, como

2 - El cambio social desde la perspectiva de Fals Borda (2009) no puede pensarse como una modificación instantánea o una transformación a nivel individual. Se trata de un proceso continuo de transformación radical y profunda de la sociedad que implica confrontar prácticas violentas y formas de opresión, explotación e injusticia históricamente configuradas. 
se profundiza más adelante, también fueron espacios propicios para diseñar acciones colectivas y para generar vínculos entre las distintas comunidades educativas.

Fase 3: análisis de la información recolectada. Toda la información recolectada en las fases previas fue registrada, transcrita (en el caso de las entrevistas), codificada y analizada con base en la propuesta de Fairclough (1995, 2011), en la que se distinguen tres niveles de análisis crítico de los discursos: 1) textual. Relativo al micro nivel del discurso en el que se describen en detalle las características de los textos objeto de análisis (estructura, léxico, registro, etc.); 2) discursivo. Relacionado con el mesonivel del discurso en el que se abordan los procesos de producción, distribución y consumo de determinados textos; 3) social. Asociado al macro nivel del discurso como práctica social, en el cual se incluye la interpretación/análisis de elementos ideológicos y políticos que construyen y a la vez son construidos por textos y prácticas discursivas de distinta naturaleza (WOODSIDE-JIRON, 2011). Si bien en la fase de análisis hubo cierta concentración del poder de la interpretación en los investigadores de la Universidad de los Andes, se generaron varios espacios de socialización de hallazgos con las comunidades educativas, las cuales retroalimentaron y fortalecieron las interpretaciones inicialmente realizadas por los investigadores de dicha Universidad.

\section{Resultados y discusión: entre el videoactivismo y el trabajo por proyectos}

\section{La Escuela Mediática: videoactivismo y ECM}

La Escuela Mediática (en adelante la Mediática) surge en el año 2000 en la ciudad de Bogotá como uno de los proyectos educativos promovido por el Colectivo de Educación Alternativa (CEAL). Desde sus inicios, la Mediática propuso una oferta educativa basada en la apropiación y producción de medios: radio, televisión, video e impresos. Se denomina Escuela Mediática, de hecho, no solo porque acoge a jóvenes que cursan la educación media, sino también porque percibe las nuevas tecnologías comunicacionales como mediaciones centrales para superar las formas tradicionales de transmisión de información, y a los maestros como mediadores entre la información que circula en distintas fuentes y canales, y la apropiación crítica, reflexiva y comprensiva que realizan los estudiantes (ESCUELA MEDIÁTICA, 2000).

Como parte del área de comunicación, los estudiantes de grado once elaboran proyectos de investigación orientados a la realización audiovisual. El proceso investigativo y creativo incorpora momentos de preproducción, producción y posproducción, articulados por una clase de comunicación que se desarrolla a lo largo del año escolar y que cuenta con una intensidad de cinco horas semanales. Según el profesor de comunicación, quien orientó el proceso en 2016 y 2017, los objetivos y fases son las siguientes:

Buscamos realizar un documental que narre alguna problemática del entorno. Hacemos todo un proceso desde las mismas bases teóricas del lenguaje audiovisual, de las teorías de la comunicación. Luego pasamos a aprender técnicas de trabajo de campo. (...) La siguiente fase 
es la investigación y el planteamiento del guión. (...) Luego viene la producción de campo. Acá los chicos están contactando y entrevistando a las fuentes. (...) En septiembre, ellos llegan con todo su material y ese mes es solo para visualizar, presentar el primer corte e ir decantando los errores en cuanto a narrativa audiovisual: es la visualización y posproducción. En octubre se trata de tenerlo finalizado para que tengamos un mes más de visibilización en otros colegios o en plataformas (Christian Plazas, comunicación personal, 6 de abril de 2017).

A partir de este proceso, en el año 2016 los estudiantes, organizados en grupos de entre cuatro y seis jóvenes, realizaron tres documentales que giraron en torno a temas cercanos a sus intereses y sensibilidades: 1) TRANSMILENIO: Opiniones sin importancia. El objetivo de este documental fue mostrar las diferentes opiniones que tienen los usuarios frente al sistema de transporte público de la ciudad de Bogotá (Transmilenio), y evidenciar cómo ellos tienen voz, pero no voto, con respecto a las necesidades del sistema y a las decisiones que se toman en torno al mismo. 2) Renovación del código de policía. El objetivo, según sus realizadores, fue promover el conocimiento de los ciudadanos acerca del nuevo Código Nacional de Policía que se aprobó en Colombia en el año 2016, y que entró en funcionamiento en 2017. 3) Conflicto armado colombiano: una guerra entre jóvenes. El propósito de este audiovisual fue generar conciencia acerca de las características y efectos del reclutamiento forzado de jóvenes en el marco del conflicto armado interno colombiano ${ }^{3}$.

Las entrevistas con exalumnos y el análisis crítico de tales documentales permitieron identificar que estos coinciden con lo que Mateos y Rajas (2014) denominan como producciones videoactivistas, en tanto cuentan con las siguientes características: cuestionan el estado de cosas apelando a entrevistas tanto a transeúntes como a expertos en los temas o problemas abordados; expresan una intencionalidad transformadora mediante un lenguaje directo y juvenil; y se comprometen, más implícita que explícitamente, con las reivindicaciones de partidos políticos de izquierda, de colectivos antimilitaristas y del movimiento de defensa de los derechos humanos. Asimismo, pese a que los documentales se inscriben en un proceso convencional de realización audiovisual (preproducción, producción, posproducción), abordan temas que ocupan un lugar secundario en los medios de comunicación dominantes, incorporando las voces y miradas de sectores usualmente marginalizados y perseguidos en Colombia. Así lo confirma una de las exalumnas entrevistadas:

Nuestro proyecto del área de comunicación se llamó lados B de una República porque nos preguntamos ¿Qué es lo que no estamos viendo? ¿Por qué solo nos dedicamos a ver el lado A de las cosas? Quisimos mostrar lo que casi nadie escucha, como el lado B de un casete. Queríamos visibilizar esas problemáticas y compartir nuestros documentales en las redes para que esas otras historias y voces fueran escuchadas en muchas partes. (Exalumna de 18 años, comunicación personal, 19 de septiembre de 2017).

Dicha intención es especialmente evidente en el documental Conflicto armado colombiano: una guerra entre jóvenes, en el que se involucran las voces de movimientos

3- Las producciones audiovisuales de los estudiantes de la Escuela Mediática se pueden consultar en: goo.gl/uQAx8z 
como la Unión Patriótica (UP), partido político que el Estado colombiano, junto con grupos paramilitares, intentaron exterminar a través del asesinato selectivo de sus militantes: desde mediados de los años ochenta y durante los últimos treinta años, alrededor de 5.000 militantes de este movimiento fueron asesinados. Esta persecución tuvo eco en los medios de comunicación dominantes en Colombia. Como se recuerda, frente al genocidio político del que fueron víctimas los militantes de este movimiento, los grandes medios de comunicación colombianos que pertenecen a los dos grupos económicos más poderosos del país (grupo Santo Domingo y grupo Ardila Lule), en ocasiones guardaron un silencio cómplice, otras veces justificaron los asesinatos bajo la falsa idea de que "[...] todos eran integrantes de la guerrilla de las FARC", y otras veces más difundieron titulares sensacionalistas: "[...] uno más de sus miembros asesinados" (GAVIRIA, 2015, p. 5). Por el contrario, el documental mencionado incluye fragmentos de una entrevista realizada a la presidenta de este partido político, quien expresa sus opiniones acerca del conflicto armado, la objeción de conciencia y la persecución política que ha padecido la UP. Ello permite confrontar las representaciones dominantes sobre la historia del conflicto armado colombiano y promover narrativas alternativas frente al mismo.

Otro aspecto para destacar es que el videoactivismo de los estudiantes de la Escuela Mediática se complementa con el uso de otras plataformas, como es el caso de YouTube. En este medio, el documental TRANSMILENIO: Opiniones sin importancia que cuenta con un mayor número de visualizaciones, da pistas importantes acerca de las características que puede tener una producción videoactivista para tener mayor incidencia o impacto en los públicos: corta duración, entrevistas a personas del común y no solo a expertos en el tema-problema abordado, y un involucramiento alto de los realizadores en el audiovisual (una de las estudiantes presenta y desarrolla, con ciertos tintes de humor, la línea narrativa del documental). Frente a este último punto, cabe señalar que, si bien "[...] existe una tendencia por parte de los colectivos videoactivistas a no formar parte de la acción frente a la cámara, es decir, a no ser la noticia sino quien la registra” (CAMUÑAS, 2014, p. 95), un involucramiento mayor de los realizadores en el audiovisual puede generar vínculos más fuertes con las audiencias y favorecer los propósitos transformadores.

Tales elementos fueron tenidos en cuenta en los talleres implementados a lo largo del 2017. En la primera sesión de este conjunto de talleres se conversó con los veinticuatro estudiantes de grado once en torno a la siguiente pregunta: ¿Quién es o puede ser un ciudadano del mundo? Entre las respuestas obtenidas se destacaron las siguientes: una persona que viaja, que conoce otros países y que no tiene dificultades para ir más allá de las fronteras; una persona que maneja otras lenguas; personas que tienen ciertos conocimientos relacionados con lo que pasa en el mundo y con la diversidad cultural; personas que no pertenecen solo a un lugar sino a varios lugares, es decir, al mundo. En un segundo momento se identificaron aquellas problemáticas que según los jóvenes afectan más a la humanidad en la actualidad, entre las que se destacaron las relacionadas con el medio ambiente, las violencias, la intolerancia y la globalización que homogeniza las culturas. Para terminar, se conversó con el grupo acerca de los temas que ya se encontraban investigando como parte del proceso de realización audiovisual, y se les 
preguntó de qué manera estos podrían contribuir a la ECM, frente a lo cual respondieron que lo podrían hacer a través de la generación de conciencia.

Los siguientes talleres giraron en torno a las posibilidades del videoactivismo para confrontar algunas de estas problemáticas y lograr aquella generación de conciencia. También buscaron complementar el proceso de realización audiovisual que ya adelantaban en la clase de comunicación llamando la atención sobre algunos de los aspectos, ya mencionados, derivados del análisis de los documentales del año 2016. Al final de 2017 el resultado fueron cinco documentales, tres vinculados con temáticas ambientales, uno acerca de los estereotipos de género excluyentes y otro sobre la diversidad artística y cultural de la ciudad de Bogotá. Todos estos documentales son de corta duración (menos de 15 minutos), involucran de forma explícita a sus realizadores y visibilizan posturas alternativas a las que circulan por los medios de comunicación dominantes en Colombia. A propósito, especial mención merece el documental La voz de un pueblo, cuyo objetivo, en palabras de los jóvenes realizadores, fue el siguiente:

Queríamos mostrar el sentir del pueblo de Pasca [municipio ubicado en el departamento de Cundinamarca, a $86 \mathrm{KM}$ de la ciudad de Bogotá] cuando se abre la posibilidad de que lleguen empresas a hacer exploración y explotación petrolera en su territorio, que es muy cercano al páramo del Sumapaz (...) queríamos que el documental hablara no desde los expertos sino desde la comunidad. (Estudiante de 18 años de la Mediática, comunicación personal, 10 de noviembre de 2017).

Imagen 1- Póster promocional del documental La voz de un pueblo

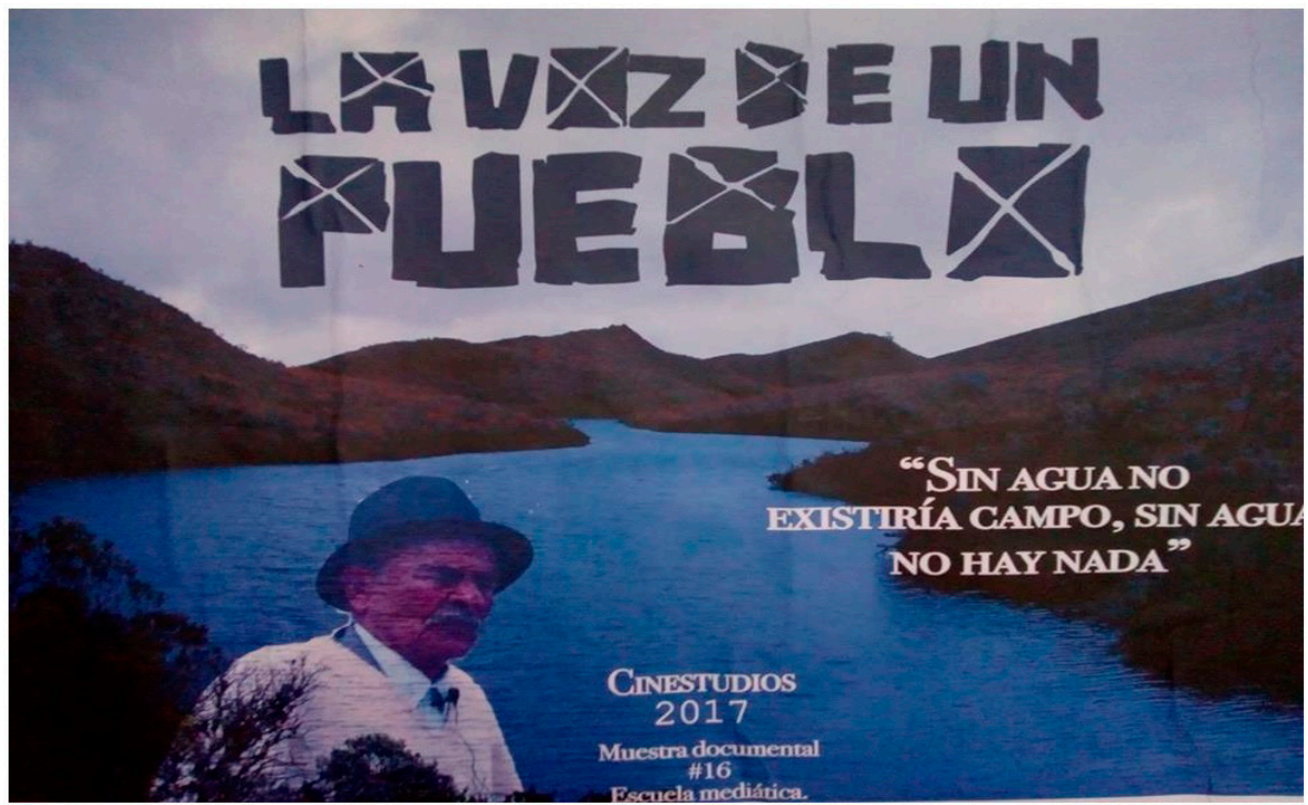

Fuente: Elaboración de estudiantes de la Mediática, 2017. 
Esta intención, que confirma el cariz videoactivista de las producciones audiovisuales de los estudiantes de la Mediática, se evidencia a través del protagonismo dado en el documental a las voces de los campesinos, quienes destacan los efectos negativos, sociales y ambientales, que la industria petrolera puede generar en su municipio. No menos importante es el hecho de que, según los jóvenes realizadores, su documental logra aportar a la ECM. Así lo explicó uno de los estudiantes:

La ciudadanía mundial no puede ser algo que se quede en las urbes, en las grandes urbes, en Bogotá, en Medellín, en Cali, sino que también debemos pensarnos desde el campo, para entenderlo y cuidarlo. La identidad campesina se está fortaleciendo y esto ayuda un poco a la ciudadanía mundial, que no tiene que ver solo con globalización o con grandes ciudades. El páramo del Sumapaz y tantas riquezas que tiene este país, no son solo nuestras como colombianos, sino también son el patrimonio y el futuro de todo el planeta, por eso debemos protegerlos. (Estudiante de 18 años de la Escuela Mediática, comunicación personal, 10 de noviembre de 2017).

De esta forma, los documentales producidos en 2017 aportan a la ECM no solo de los realizadores, quienes se familiarizaron con el concepto y lo llenaron de contenido desde sus propios saberes, sino también de aquellos públicos a los que lograron impactar. En efecto, tales documentales de la Mediática fueron presentados a padres y madres de familia de los estudiantes realizadores en una muestra documental, así como también a los estudiantes y docentes del Colegio Venecia en un evento de intercambio y socialización de experiencias que tuvo lugar en la Universidad de los Andes de Bogotá. En estos eventos fue claro lo que significó la ECM para los jóvenes de grado once de la Mediática: una forma de generar conciencia y reflexión en torno a temas que conciernen a la humanidad y que son muy cercanos a las sensibilidades juveniles, como el cuidado del medio ambiente.

\section{El Colegio Venecia: trabajo por proyectos y activismo mediado por TIC}

El Colegio Venecia es una Institución educativa oficial que en su área de Educación Media Integral (EMI) cuenta con una oferta educativa basada en cuatro asignaturas: Gestión de Proyectos, Técnicas de Comunicación, Medios Audiovisuales-Multimedia y Programación. Los estudiantes que pasan por el área deben elaborar en parejas un proyecto en torno a problemáticas de su contexto, que identifiquen y quieran aportar a resolver. La clase de gestión de proyectos es la columna vertebral del proceso. En esta clase los estudiantes elaboran encuestas para identificar problemas o necesidades de sus contextos cercanos, diseñan el marco legal y referencial de su proyecto, proponen objetivos y preguntas de investigación, entablan vínculos con organizaciones sociales que puedan expandir sus iniciativas y elaboran propuestas de intervención socioeducativa que incluyen talleres dirigidos a otros estudiantes y el diseño de alguna mediación virtual o tecnológica: páginas web, aplicaciones, revistas y juegos virtuales, entre otros. Para consolidar esta última parte de su propuesta, a lo largo del año reciben acompañamiento y orientación por parte de los docentes de las demás asignaturas del área. 
Como parte de la IAP del año 2017 los profesores de Gestión de Proyectos pidieron a los estudiantes que ampliaran la mirada y que investigaran, de cara a la construcción de su marco referencial, noticias no solo locales sino también nacionales y mundiales relacionadas con los temas de sus proyectos de investigación. Los temas de los proyectos, a su vez, estuvieron relacionados con problemáticas ambientales, educativas o con algún tipo de violencia, como el cyberbullying o el desplazamiento forzado. Uno de los proyectos, por ejemplo, buscó acompañar el proceso formativo de niños y niñas desplazados que llegan del campo a la ciudad, muchos de ellos víctimas de la violencia en Colombia. Los dos estudiantes que lideraron la iniciativa lograron el apoyo de una corporación de trabajo comunitario denominada Inti Tekoa, con quienes desarrollaron conjuntamente una propuesta formativa basada en el trabajo colectivo, el uso de herramientas tecnológicas y las estrategias pedagógicas propias de la educación popular.

Autores como Standish (2014) han destacado que una de las limitaciones de la ECM tiene que ver con que las actividades que en nombre de esta se promueven suelen estar diseñadas dentro de una perspectiva ética particular (liberal, occidental) que en última instancia está cobijada por el marco individualista dominante. Por tanto, las acciones solo apuntan a cambiar el comportamiento individual en respuesta a normas establecidas, pero no comprometen a los individuos en proyectos colectivos para el cambio social. Sin embargo, el trabajo por proyectos que tuvo lugar en el colegio Venecia no reprodujo dicha limitación, pues surgió de las necesidades identificadas por los jóvenes en sus contextos cercanos y favoreció el establecimiento de alianzas con organizaciones comunitarias que dotaron las iniciativas estudiantiles y los aprendizajes que de estas se derivaron, de un carácter mucho más colectivo. Así lo expresó uno de los estudiantes cuando se le preguntó acerca de los aprendizajes que le dejó el proyecto y sus vínculos con la ECM:

Aprendimos a trabajar en equipo teniendo en cuenta cada punto de vista de los integrantes para fortalecer nuestras ideas. También aprendimos a ser profesores, a liderar grupos y a compartir nuestros conocimientos. Considero que nuestro proyecto aporta a la ECM porque estamos en un periodo de la historia en el que el desplazamiento es un gran problema que afecta a muchos países alrededor del mundo. Al fortalecer los procesos de enseñanza/aprendizaje de niños de 7 a 12 años que llegaron desplazados a Bogotá, contribuimos a solucionar un poco la situación de ellos y sus familias en el lugar al que deben llegar, por consecuencia de la Violencia. (Estudiante de 17 años del Colegio Venecia, Comunicación Personal, 30 de noviembre de 2017).

Ahora bien, en los talleres en los que se reflexionó sobre ciudadanía mundial y sobre las posibilidades del activismo mediado por tecnologías digitales para promoverla, los estudiantes asociaron la ciudadanía mundial al intercambio de experiencias y a la generación de vínculos con otras comunidades educativas de Colombia y del mundo. Por esta razón, con el propósito de construir ciudadanía mundial, encontraron grandes oportunidades en el uso de redes y plataformas virtuales, de manera que se aventuraron a diseñar un espacio web, un correo electrónico (ciudadanosdelmundo07@gmail.com), una cuenta en Twitter (@ciudadanos_M07), una página en Facebook denominada Ciudadanos Del Mundo (disponible en goo.gl/QEBaCR), entre otras plataformas. Por estos espacios 
circularon fotos, videos, infografías y demás contenidos gráficos y audiovisuales elaborados por los estudiantes de ambos colegios, incluidos los documentales de la Escuela Mediática.

Estos espacios permitieron el intercambio de contenidos, el reconocimiento mutuo de las comunidades educativas de ambos colegios y la generación de un sentido de pertenencia al mundo y, por tanto, un sentido de identificación en tanto ciudadanos del mundo. De igual forma, los estudiantes del Colegio Venecia propusieron un logo que presentaron a los estudiantes de la Escuela Mediática, en uno de los talleres conjuntos en los que tuvieron la posibilidad de conocerse e intercambiar ideas. Asimismo, el logo fue incluido como imagen de perfil en los distintos espacios virtuales. La siguiente es la explicación que acompaña el logo en redes sociales:

Somos estudiantes del Colegio Venecia. Desarrollamos la siguiente imagen del proyecto "Ciudadanos Del Mundo" tomando como base la solución a una problemática que estamos enfrentando globalmente: la discriminación. Con esta imagen queremos reflejar un símbolo de unión y solidaridad, donde la misión sea afrontar este problema con unión e igualdad como ciudadanos del mundo que somos. Reflejamos una parte de la diversidad de nuestro mundo, con el fin de eliminar el pensamiento racial, en torno a la discriminación tanto cultural, económica, etc... El mundo está representado en forma de corazón como demostración de unión y amor, todo esto sobre dos manos que arropan al mundo tanto como a sus ciudadanos. ¿Cómo les parece?

\section{Imagen 2- Logo Ciudadanos del Mundo}

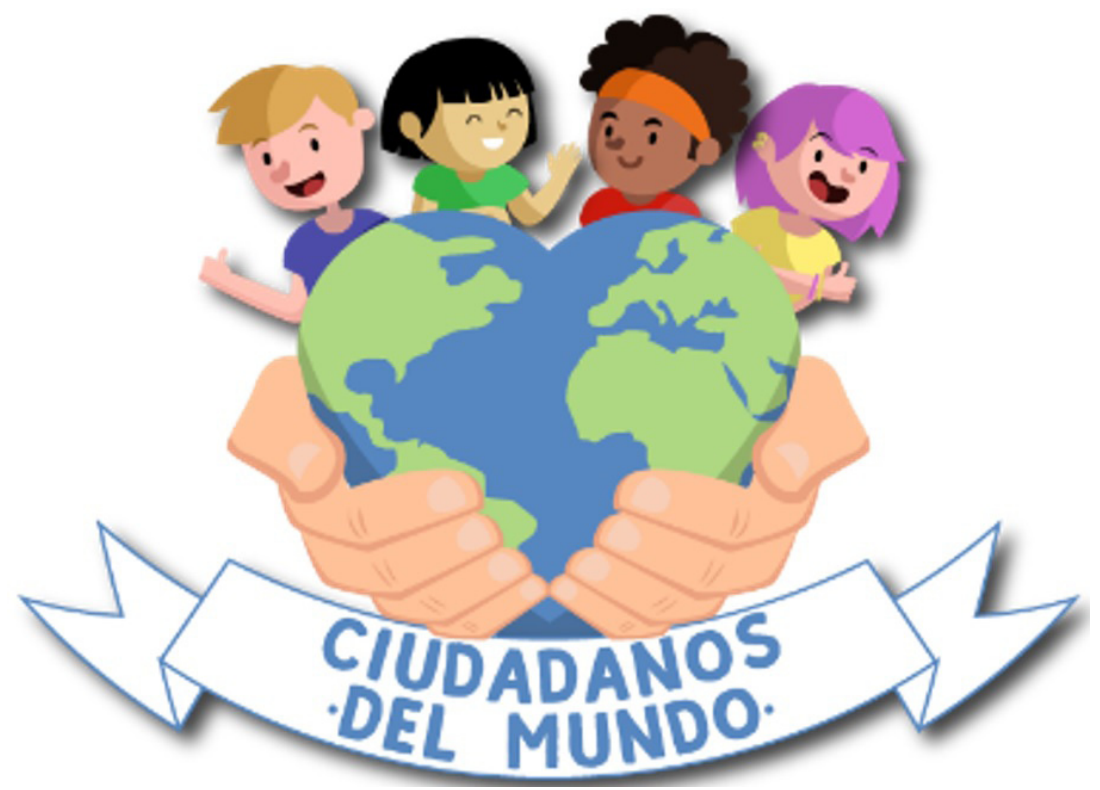

Fuente: Elaboración de estudiantes del Colegio Venecia, 2017.

Las plataformas y espacios virtuales creados por los estudiantes del Colegio Venecia también propiciaron el activismo mediado por herramientas tecnológicas. Los estudiantes 
subieron especialmente a la página de Facebook fotos, videos e infografías relacionadas con problemáticas que consideraron crucial denunciar e intervenir en la actualidad, como la contaminación, el racismo, la pobreza, las guerras, entre otras. De esta forma, a través del trabajo por proyectos y de una apropiación crítica de plataformas virtuales, demostraron que lo mundial de la ciudadanía mundial no tiene que ver solamente con el acceso a información internacional, con el manejo de lenguas extranjeras o con conocimientos que desbordan la escala local. Tiene que ver también con las posibilidades de entablar vínculos con otros y de generar pequeñas acciones que propicien reflexiones y motiven la movilización en torno a temas que afectan a la humanidad en su conjunto. Esto es lo que intentaron y continúan intentando hacer los estudiantes del Colegio Venecia a través del uso de herramientas tecnológicas y del trabajo por proyectos.

\section{Conclusiones}

Entre los múltiples estudios que analizan estrategias de ECM en el ámbito de la educación formal y en diferentes contextos nacionales e internacionales (JOHNSON; BOYER; BROWN, 2011; PERAZA, 2016; ENGEL; FUNDALINSKI; CANNON, 2016; AKTAS et al., 2017; RAPOPORT, 2013), pocos enfatizan en la producción audiovisual y ninguno en temas de videoactivismo. El presente estudio evidencia un vínculo posible entre el videoactivismo y la ECM que puede continuar explorándose en próximos trabajos. Si la ECM tiene que ver con el empoderamiento de los sujetos de cara a la construcción de un mundo más “[...] justo, pacífico, tolerante, inclusivo, seguro y sostenible” (UNESCO, 2016, p. 16), la desestabilización de las estructuras de poder y representación tradicionales deviene estratégica, elemento que constituye uno de los rasgos característicos del videoactivismo (ASKANIUS, 2013).

No en vano el videoactivismo se ha relacionado con la creación de una nueva esfera pública, en tanto permite abrir espacios a perspectivas alternativas que disputan el poder simbólico que tienen los medios dominantes para definir/producir los acontecimientos del mundo social (MATEOS; RAJAS, 2014). Como explica García (2014), aunque las temáticas relacionadas con el videoactivismo pueden ser muy amplias, toda producción videoactivista se orienta a la transformación y para ello, tiende a visibilizar realidades ocultas o silenciadas por los medios de comunicación de masa. Buena parte de ello se pudo constatar en el trabajo adelantado en la Escuela Mediática, en donde los estudiantes de grado once por medio de proyectos colectivos de investigación, realizaron documentales en los que muestran acontecimientos o problemas que usualmente no son presentados por los medios de comunicación dominantes, incorporando voces de sectores tradicionalmente subalternizados en Colombia. Ello contribuye a la ECM, pues como señala Standish (2014) la ECM ha sido pensada como la posibilidad de promover una educación más inclusiva en la que las voces y perspectivas de comunidades marginadas y de las minorías, no solo a nivel nacional sino global, tengan cabida.

De igual forma, los documentales de los estudiantes de la Mediática impulsan la generación de conciencia en torno a temas que conciernen a la humanidad, como el cuidado del medio ambiente. Esto lo hacen a través de proyectos de investigación en los 
que la comunicación social crítica ocupa un lugar protagónico. Tal elemento lo comparten con los estudiantes del Colegio Venecia, quienes aportan a la ECM desde la creación de espacios virtuales con los que buscan generar sentido de pertenencia al mundo y propiciar escenarios de intercambio y aprendizaje mutuo entre comunidades educativas ubicadas en localidades geográficamente distantes. Lo anterior lo logran por medio del desarrollo de proyectos que, al igual que en la Mediática, propician experiencias alternativas de aprendizaje que confrontan incluso las lógicas de la escuela tradicional.

En efecto, mientras a la escuela tradicional se asiste a recibir información sobre contenidos curriculares y disciplinas definidas, a la Mediática y al área de EMI del Colegio Venecia se acude a participar en la construcción de proyectos altamente significativos para los sujetos, relacionados con sus necesidades, expectativas e intereses. De igual forma, a diferencia de los currículos inflexibles y de los contenidos que los sistemas educativos en ocasiones buscan imponer desde arriba para que caigan a la escuela de una manera prescriptiva, los proyectos nacen desde abajo (IEIE, 2017), desde los contextos locales, y es desde ahí desde donde los jóvenes se aventuran a pensar en contextos más amplios que desbordan lo local y lo nacional. En definitiva, el videoactivismo y el trabajo por proyectos ofrecen pistas relevantes acerca de lo que puede llegar a ser, o ya estar siendo, una ECM que permita cuestionar formas de poder y representación dominantes, entablar vínculos y nuevas formas de identificación aprovechando las herramientas digitales, generar comprensiones situadas incorporando perspectivas alternativas frente a determinados problemas y construir, desde los contextos locales, "[...] formas más éticas y profundas de relacionarnos con los otros y con el mundo” (ANDREOTTI; PASBY, 2013, p. 435).

Para terminar, se puede afirmar que con esta investigación se logró aportar al campo educativo en varias direcciones. A nivel temático se promovió la ECM como un campo de reflexión y análisis que aún no ha sido lo suficientemente abordado en América Latina, y que requiere de aproximaciones empíricas que superen el énfasis teórico predominante en la literatura especializada (GOREN; YEMINI 2018). A nivel teórico-político se reivindica el enfoque decolonial en la investigación educativa y se ilustra cómo este se puede materializar en estrategias pedagógicas concretas, ligadas, en este caso, al videoactivismo y el trabajo por proyectos. Por último, a nivel metodológico-epistemológico se revaloran las formas de investigación participativa que rompen con la distinción sujeto/objeto y tienen como horizonte ético y político la solidaridad, el diálogo y el trabajo colaborativo.

Así, ante "[...] la imagen (particularmente acentuada en el nivel de la experiencia doctoral) del académico aislado construyendo laboriosamente un marco a partir de literaturas que ha dominado por sí mismo" (ESCOBAR, 2010, p. 10), este estudio se desarrolló desde las experiencias educativas, no sobre ellas, y reconociendo a sus actores como agentes generadores de significados, iniciativas y conocimientos que pueden aportar tanto a la reflexión teórica como a la transformación colectiva de los contextos locales/globales que hoy nos convocan a la acción. Sin duda, dicha transformación pasa por el trabajo colaborativo, por la pedagogía proyectiva, por la apropiación crítica de herramientas tecnológicas y por los intereses y sensibilidades juveniles. Esto es lo que nos 
enseñó la experiencia de investigación/intervención en la Escuela Mediática y el Colegio Venecia, y lo que nos motiva a seguir trabajando en la misma dirección.

\section{Referencias}

AKTAS, Fatih et al. Institutionalizing global citizenship: a critical analysis of higher education programs and curricula. Journal of Studies in International Education, Amsterdam, v. 21, n. 1, p. 65-80, 2017.

ANDREOTTI, Vanessa. Soft versus critical global citizenship education. Policy \& Practice, Belfast, n. 3, p. 40-51, 2006.

ANDREOTTI, Vanessa. (Towards) decoloniality and diversality in global citizenship education. Globalisation, Societies and Education, Bristol, v. 9, n. 3-4, p. 381-397, 2011.

ANDREOTTI, Vanessa; PASHBY, Karen. Digital democracy and global citizenship education: mutually compatible or mutually complicit? The Educational Forum, London, v. 77, n. 4, p. 422-437, 2013.

ASKANIUS, Tina. Online video activism and political mash-up genres. JOMEC Journal Journalism Media and Cultural Studies, London, v. 1, n. 4, p. 1-21, 2013.

BALARIN, Maria. Global citizenship and marginalisation: contributions towards a political economy of global citizenship. Globalisation, Societies and Education, Bristol, v. 9, n. 3-4, p. 355-366, 2011.

BONI, Alejandra; HOFMANN-PINILLA, Amparo; SOW, Jadicha. Educando para la ciudadanía global: una experiencia de investigación cooperativa entre docentes y profesionales de las ONGD. Estudios Sobre Educación, Navarra, v. 23, n. 1, p. 63-81, 2012.

CAMICIA, Steven; FRANKLIN, Barry. What type of global community and citizenship? Tangled discourses of neoliberalism and critical democracy in curriculum and its reform. Globalisation, Societies and Education, Bristol, v. 9, n. 3-4, p. 311-322, 2011.

CAMUÑAS, Manuel. Miradas colectivas: veinte años de videoactivismo en España. In: MATEOS, Concha; RAJAS, Mario (Org.). Videoactivismo: acción política cámara en mano. La Laguna: Latina, 2014. p. 7-98.

CATALANO, Theresa. Occupy: a case illustration of social movements in global citizenship education. Education, Citizenship and Social Justice, Belfast, v. 8, n. 3, p. 276-288, 2013.

CHO, Hye Seung; MOSSELSON, Jacqueline. Neoliberal practices amidst social justice orientations: global citizenship education in South Korea. Compare: a Journal of Comparative and International Education, London, v. 47, n. 4, 2017.

CHUNG, Sheng Kuan; KIRBY, Michael S. Media literacy art education: logos, culture jamming and activism. Art Education, Alexandria, v. 62, n. 1, p. 34-39, 2009

ENGEL, Laura; FUNDALINSKI, Jessica; CANNON, Tess. Global citizenship education at a local level: a 
comparative analysis of four U.S. urban districts. Revista Española de Educación Comparada, Madrid, n. 28, p. 23-51, 2016.

ENGUIX, Begonya. Activismo y prácticas digitales en la construcción de una esfera LGTB en España. Revista Dados, Rio de Janeiro, v. 59, n. 3, p. 755-787, 2016.

ESCOBAR, Arturo. Territorios de diferencia: lugar, movimientos, vida, redes. Popayán: Envión, 2010.

ESCUELA MEDIÁTICA. Proyecto educativo institucional: educación media bivalente con énfasis en la producción y recepción crítica de medios y nuevas tecnologías comunicacionales. Bogotá: Documento Inédito, 2000.

FAIRCLOUGH, Norman. Critical discourse analysis. London: Longman, 1995.

FAIRCLOUGH, Norman. Semiotic aspects of social transformation and learning. In: ROGERS, Rebecca (Org.). An introduction to critical discourse analysis in education. New York: Routledge, 2011. p 119-127.

FALS BORDA, Orlando. Experiencias teórico-prácticas. In: MONCAYO, Víctor (Org.). Una sociología sentipensante para América Latina. Bogotá: Clacso-Siglo del Hombre, 2009. p. 303-363.

FULLAM, Jordan. Becoming a youth activist in the internet age: a case study on social media activism and identity development. International Journal of Qualitative Studies In Education, Indianapolis, v. 30, n. 4, p. 406-422, 2017.

GARCÍA, Paloma. Videoactivismo de convocatoria: el lenguaje publicitario en las mareas ciudadanas. In: MATEOS, Concha; RAJAS, Mario (Org.). Videoactivismo: acción política cámara en mano. La Laguna: Latina, 2014. p. 135-166.

GAVIRIA, Alejandra. UP: imágenes de un sueño. [Introducción]. In: GAVIRIA, Alejandra (Org.). Unión patriótica: imágenes de un sueño. Bogotá: Comité Permanente por la Defensa de los Derechos Humanos, 2015. p 5-9.

GLENN, Cerise. Activism or "Slacktivism?": digital media and organizing for social change. Communication Teacher, London, v. 29, n. 2, p. 81-85, 2015.

GONZÁLEZ-LIZARRAGA, Guadalupe; BECERRA-TRAVER, María T.; YANEZ-DIAZ, Mireya. Ciberactivismo: nueva forma de participación para estudiantes universitarios. Comunicar, Huelva, v. 46, n. 24, p. 47-54, 2016.

GOREN, Heela; YEMINI, Miri. Obstacles and opportunities for global citizenship education under intractable conflict: the case of Israel. Compare: a Journal of Comparative and International Education, London, v. 48, n. 3, p. 397-413, 2018.

IEIE - Instituto de Estudios e Investigaciones Educativas. Conclusiones del cuarto encuentro de experiencias alternativas en educación. In: AGUILAR, Juan (Org.). Experiencias alternativas en educación: memorias del cuarto encuentro. Bogotá: IEIE, 2017. p. 407-414. 
JOHNSON, Paula; BOYER, Mark; BROWN, Scott. Vital interests: cultivating global competence in the international studies classroom. Globalisation, Societies and Education, Bristol, v. 9, n. 3-4, p. 503-519, 2011.

JOOSTE, Nico; HELETA, Savo. Global citizenship versus globally competent graduates: a critical view from the south. Journal of Studies in International Education, Amsterdam, v. 21, n. 1, p. 39-51, 2017.

LEÓN, Martín. Construir saberes desde la diversidad para disfrutar y aprender: Colegio Venecia I.E.D. In: AGUILAR, Juan (Org.). Experiencias alternativas en educación: memorias del cuarto encuentro. Bogotá: IEIE, 2017. p. 283-292.

LINDER, Chris et al. From margins to mainstream: social media as a tool for campus sexual violence activism. Journal of Diversity in Higher Education, Fort Lauderdale, v. 9, n. 3, p. 231-244, 2016.

MARSHALL, Harriet. Instrumentalism, ideals and imaginaries: theorising the contested space of global citizenship education in schools. Globalisation, Societies and Education, Bristol, v. 9, n. 3-4, p. 411-426, 2011.

MATEOS, Concha; RAJAS, Mario. Videoactivismo: concepto y rasgos. In: MATEOS, Concha; RAJAS, Mario (Org.). Videoactivismo: acción política cámara en mano. La Laguna: Latina, 2014. p. 15-34.

NIETO, Diego. Citizenship education discourses in Latin America: multilateral institutions and the decolonial challenge. Compare: a Journal of Comparative and International Education, London, v. 47, n. 6, p. 1-19, 2017.

PAIS, Alaexandre; COSTA, Marta. An ideology critique of global citizenship education. Critical Studies in Education, London, v. 58, n. 1, p. 1-16, 2017.

PEÑA, Patricia; RODRÍGUEZ, Raúl; SÁEZ, Chiara. Movimiento estudiantil en Chile, aprendizaje situado y activismo digital: compromiso, cambio social y usos tecnológicos adolescentes. OBETS, Alicante, v. 11, n. 1, p. 287-310, 2016.

PERAZA, Cecilia. Interpretaciones de la educación para la ciudadanía global en la reforma de la educación media superior en México. Revista Española de Educación Comparada, Madrid, n. 28, p. 135-159, 2016.

RAPOPORT, Anatoli. Global citizenship themes in the social studies classroom: teaching devices and teachers' attitudes. The Educational Forum, London, v. 77, n. 4, p. 407-420, 2013.

REILLY, Jacqueline; NIENS, Ulrike. Global citizenship as education for peacebuilding in a divided society: structural and contextual constraints on the development of critical dialogic discourse in schools. Compare: a Journal of Comparative and International Education, , Londons, v. 44, n. 1, p. 53-76, 2014.

ROMAN, Marcela; MURILLO, Javier. Disponibilidad y uso de TIC en escuelas latinoamericanas: incidencia en el rendimiento escolar. Educação e Pesquisa, São Paulo, v. 40, n. 4, p. 869-895, 2014.

SANTOS, Boaventura de Sousa. Para descolonizar el occidente: más allá del pensamiento abismal. Buenos Aires: CLACSO, 2010. 
SHULTZ, Lynette. Educating for global citizenship: conflicting agendas and understandings. Alberta Journal of Educational Research, Alberta, n. 53, p. 248-258, 2007.

STANDISH, Alex. What is global education and where is it taking us? The Curriculum Journal, London, v. 25, n. 2, p. 166-186, 2014.

TRUONG-WHITE, Hoa; MCLEAN, Lorna. Digital storytelling for transformative global citizenship education. Canadian Journal of Education, Ottawa, v. 38, n. 2, p. 1-29, 2015.

UNESCO. Educación para la ciudadanía mundial: preparar a los educandos para los retos del siglo XXl. Paris: Unesco, 2016.

VALENZUELA, Sebastián. Unpacking the use of social media for protest behavior: the roles of information, opinion expression, and activism. American Behavioral Scientist, Sedona, v. 57, n. 7, p. 920-942, 2013.

VEUGELERS, Weil. Creating critical-democratic citizenship education: empowering humanity and democracy in Dutch education. Compare, London, v. 37, n. 1, p. 105-119, 2007.

WATSON, Sam. An exploration into the teaching of cosmopolitan ideals: the case of 'global citizenship'. Citizenship, Social and Economic Education, London, v. 12, n. 2, p. 110-117, 2013.

WOODSIDE-JIRON, Haley. Language, power, and participation: using critical discourse analysis to make sense of public policy. In: ROGERS, Rebecca (Org.). An introduction to critical discourse analysis in education. New York: Roudledge, 2011. p. 154-182.

Recibido en: 31.01 .2018

Revisiones en: 04.04.2018

Aprobado en: 16.05.2018

Nicolás J. C. Aguilar Forero es doctor en Ciencias Sociales, magíster en Estudios Culturales, antropólogo y profesional en Lenguajes y Estudios Culturales. Hasta enero de 2019 se desempeñó como investigador posdoctoral de la Facultad de Educación de la Universidad de los Andes. 